OPEN ACCESS

Edited by:

Hui Xia,

Shanghai Agrobiological Gene Center,

China

Reviewed by:

Hamid Khazaei,

University of Saskatchewan, Canada

Hongjun Liu,

Shandong Agricultural University,

China

${ }^{*}$ Correspondence:

Jianlong Xu

xujlcaas@126.com

Zhikang Li

zhkli1953@126.com

†These authors have contributed equally to this work.

Specialty section:

This article was submitted to

Plant Breeding,

a section of the journal

Frontiers in Plant Science

Received: 04 December 2017

Accepted: 27 February 2018

Published: 15 March 2018

Citation:

Cui Y, Zhang $W, L$ in $X, X u S, X U J$ and

Li Z (2018) Simultaneous

Improvement and Genetic Dissection

of Drought Tolerance Using Selected

Breeding Populations of Rice.

Front. Plant Sci. 9:320

doi: 10.3389/fpls.2018.00320

\section{Simultaneous Improvement and Genetic Dissection of Drought Tolerance Using Selected Breeding Populations of Rice}

\author{
Yanru Cui ${ }^{1 \dagger}$, Wenying Zhang ${ }^{2 \dagger}$, Xiuyun Lin $^{3}$, Shizhong $X u^{4}$, Jianlong $X u^{1,5 *}$ and \\ Zhikang $L i^{1,5 *}$
}

\begin{abstract}
'Institute of Crop Sciences, National Key Facility for Crop Gene Resources and Genetic Improvement, Chinese Academy of Agricultural Sciences, Beijing, China, ${ }^{2}$ Ningxia Academy of Agriculture and Forestry Sciences, Yinchuan, China, ${ }^{3}$ Rice Research Institute, Jilin Academy of Agricultural Sciences, Changchun, China, ${ }^{4}$ Department of Botany and Plant Sciences, University of California, Riverside, CA, United States, ${ }^{5}$ Shenzhen Institute of Breeding and Innovation, Chinese Academy of Agricultural Sciences, Shenzhen, China
\end{abstract}

Drought is the most important factor limiting rice yield in most rainfed areas of Asia and Africa. Four large $\mathrm{BC}_{2} \mathrm{~F}_{2}$ populations consisted of 3,200 individuals, which were derived from crosses between an elite Geng variety, Jigeng88, and four donors from three different countries, were screened and progeny tested under severe drought stress, resulting in the development of 72 introgression lines (ILs) with significantly improved yield compared to the recurrent parent Jigeng88. These DT ILs plus four random populations (without drought selection population) from the same crosses were evaluated in replicated trials under both drought stress and non-stress conditions in two environments, and characterized with simple sequence repeat (SSR) markers to understand how directional selection was operating on the genetic variation of DT of rice. Thirteen DT QTLs of large effect were identified based on the significant allelic and genotypic frequency shits in the DT ILs by using the joint segregation distortion method. The 13 QTLs were validated by the genotypic differences at individual QTL in the random populations. Putative genetic networks consisting of 30 loci in 29 functional genetic units underlying DT were detected by $X^{2}$ tests and non-random associations between or among DT loci in DT ILs from the four populations. Most large-effect DT QTLs were previously reported and located in the upstream of the genetic networks as putative regulators, and were either mapped to important regulatory genes for DT or drought responsiveness reported previously. In our study, five promising ILs with significantly improved yield were selected under both drought and normal irrigated conditions. The QTLs and their genetic networks underlying DT detected provided useful genetic information for further improving DT and yield using designed QTL pyramiding.

Keywords: QTL, drought tolerance, functional genetic units, non-random associations, hidden genetic diversity 


\section{INTRODUCTION}

Rice (Oryza sativa L.) is the major food crop for more than 700 million people and more than $90 \%$ of rice in the world is grown and consumed in Asia (Ji et al., 2012; Palanog et al., 2014). However, with the deterioration of environment, area with severe water shortage is expected to increase. In Asia, about 50\% of the rice land is rainfed rice area where the water supply is unpredictable and droughts are common. Rice is sensitive to drought stress at the reproductive stage, when a slight drought stress can cause drastic yield loss (Kamoshita et al., 2008; Palanog et al., 2014). Drought has been a major abiotic stress factor for limiting rice production in rainfed ecosystem. Developing drought tolerance (DT) rice cultivars is the direct and effective way to reduce crop loss.

Developing DT rice varieties is often challenging because of the complexity of DT. In nature, drought stress may occur at any stage of rice growth, and the effect of drought stress on rice are various at different growth stage. The rice species has 12 chromosomes with the whole genome size of $430 \mathrm{Mb}$. In rice breeding, direct selection for improving DT was often ineffective because rice DT often show a considerable degree of genotype by environment interaction (Fukai and Cooper, 1995). As a result, tremendous efforts have been devoted to genetically dissect DT related secondary traits such as root architecture, leaf water potential and relative water content, etc. Unfortunately, indirect selection for secondary traits have not been effective to improve DT in rice due to poor correlation between the secondary traits and grain yield under drought stress (Palanog et al., 2014). Recent studies showed that direct selection for grain yield (GY) in artificial or natural drought stress conditions is the most effective way for developing DT rice with high yield potential under nontress conditions (Venuprasad et al., 2007, 2008; Guan et al., 2010). However, it often takes long time for cultivar development using traditional methods of rice breeding on the basis of simple cross making and phenotypic selection. An alternative method of improving breeding effectiveness is to identify quantitative trait loci (QTLs) with large and consistent effects in different populations under drought stress conditions. The identified QTL can be used for marker assistant breeding (MAB) (Venuprasad et al., 2011). Thus, many efforts have been made to identify largeeffect QTL affecting DT and develop marker assisted selection systems for improving rice DT (Xu et al., 2005; Yue et al., 2005; Palanog et al., 2014). Again, despite larger numbers of QTL related to DT have been detected, relatively few of them have large and consistent effects for an efficient MAS program.

In order to fill the gap between basic genetic/molecular dissection of DT and improving DT in breeding, a new strategy has been proposed for simultaneous improvement and genetic dissection of complex traits using backcross breeding and marker-facilitated tracking of gene flow from donors to recipients from selection (Li et al., 2005b). In other words, selected breeding progeny will be used to conduct QTL mapping, which have three major advantages over the classical QTL mapping. The first one is the small size of selected breeding populations and thus requires low costs in both genotyping and phenotyping. Second, selected population often has greatly increased power in detecting QTL for the target traits under selection, but much reduced power in detecting QTL for non-target traits. The third and most important one is that mapping QTL using selected population is part of breeding and lines from selected populations are expected to carry beneficial alleles of QTL. Thus, selected lines can potentially become new varieties, but more likely can be used directly as parents in making crosses of "designed QTL pyramiding," an important step toward breeding by design (Ali et al., 2017).

In this study, we demonstrated again the strategy for simultaneous improvement and genetic dissection of DT of rice in the process of breeding. We reported the development of superior lines with significantly improved grain yield under both drought stress and irrigated conditions as well as providing useful breeding lines and genetic information for further improving rice yield and DT using designed QTL pyramiding.

\section{MATERIALS AND METHODS}

\section{Development of the Plant Materials}

A superior high yield Geng (japonica) variety, Jigeng88, which is an elite cultivar commercially grown in Jilin province of China, was used as the recurrent parent (RP) and four varieties collected from China, Malaysia, and IRRI as donors. These donors contained three Xian (indicas) and one Geng variety (Table 1). In the summer of 2005, Jigeng88 (JG88) was crossed with all donors to produce $F_{1} s$ on the experimental farm of Ningxia Academy of Agriculture and Forestry Sciences (NAAFS) in Yinchuan $\left(38.5^{\circ} \mathrm{N}, 106.2^{\circ} \mathrm{E}\right)$. The $\mathrm{F}_{1}$ plants were backcrossed with the $\mathrm{RP}$ to produce $\mathrm{BC}_{1} \mathrm{~F}_{1}$ population in Sanya $\left(18.3^{\circ} \mathrm{N}\right.$, $\left.109.3^{\circ} \mathrm{E}\right)$, Hainan Province of China during the winter season of 2005-2006. In the summer of 2006, 25-30 randomly selected plants from each of the $\mathrm{BC}_{1} \mathrm{~F}_{1}$ populations were backcrossed with the $\mathrm{RP}$ to produce $25-30 \mathrm{BC}_{2} \mathrm{~F}_{1}$ lines. From each of the crosses, $25 \mathrm{BC}_{2} \mathrm{~F}_{1}$ lines were planted (36 plants of each line in a single row) in 2007. Selfed seeds from individual plants of $25 \mathrm{BC}_{2} \mathrm{~F}_{1}$ lines of each cross were bulk harvested to produce a single bulk $\mathrm{BC}_{2} \mathrm{~F}_{2}$ population.

\section{The Screening of the $\mathrm{BC}_{2} \mathrm{~F}_{2}$ Bulk Populations for DT at the Reproductive Stage}

The screening of the $\mathrm{BC}_{2} \mathrm{~F}_{2}$ bulk populations was conducted on the experimental farm of NAAFS. The soil of the test field was a sandy clay. In the initial screen for DT, 800 25-day old seedlings of each $\mathrm{BC}_{2} \mathrm{~F}_{2}$ population were transplanted into a big 80 -row plot with 10 plants per row and a spacing of $20 \times 25 \mathrm{~cm}$ between plants and rows flanked by two rows of the RP in the summer of 2008 at the NAAFS. Figure 1A shows the field screening of drought tolerance (DT) at reproductive stage. The field was managed under the normal irrigation until the peak tillering stage 50 days after transplanting. Then, the field was drained. Flush irrigation was applied twice when drought stress became very severe due to large evaporation capacity, at an interval of 10 days (total water applied $1,600 \mathrm{~m}^{3} \mathrm{ha}^{-1}$ ), to create severe water stress at the reproductive stage. The resulting soil water 
TABLE 1 | The information of 4 rice backcross populations used for improving drought tolerance in this study.

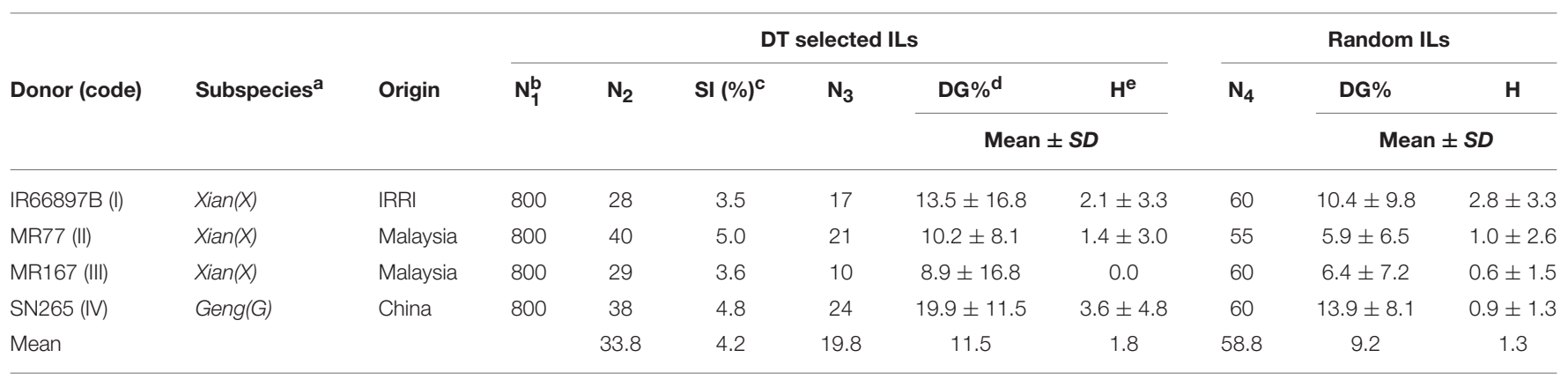

a Subspecies $X=$ Xian (indica) and $G=$ Geng (japonica).

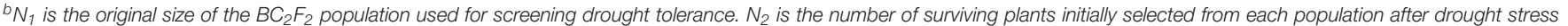

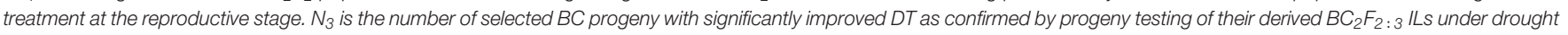
stress at the reproductive stage. $N_{4}$ is the size of each of the $B C_{2} F_{2}$ random populations used for validation.

${ }^{C} S$ I is the initial selection intensity (proportion of plants selected) in the $B C_{2} F_{2}$ populations.

${ }^{d}$ Percentage of the donor genome of the $\mathrm{BC}_{2} \mathrm{~F}_{2}$ ILs was calculated based all polymorphic SSR markers used in genotyping.

${ }^{\mathrm{e}} \mathrm{H}$, heterozygosity.

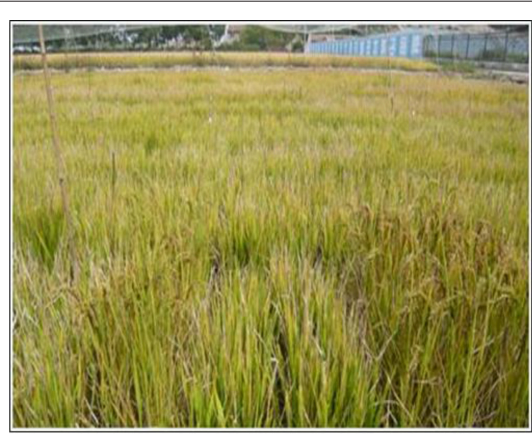

A

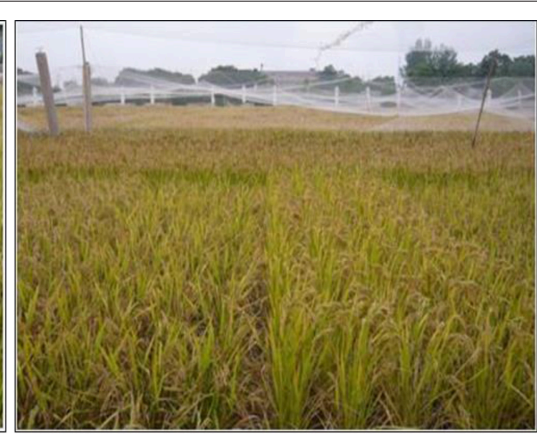

B

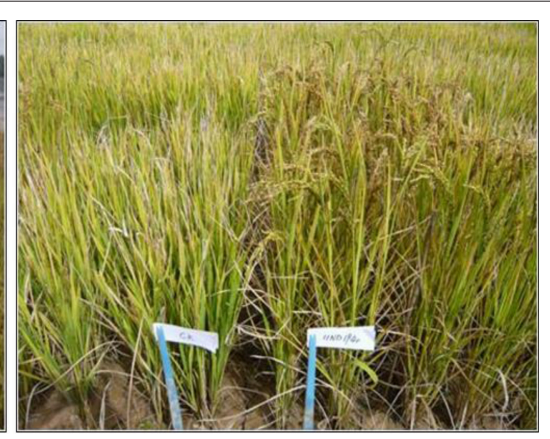

C

FIGURE 1 | Field screening and performance of drought tolerance under drought stress. (A) The field screening of DT at the reproductive stage. (B) Performance of the DT ILs under drought stress. (C) Comparison of DT IL and JG88 under drought stress (The left side are JG88 and right side are DT ILs).

content was $\sim 16-19 \%(\mathrm{v} / \mathrm{v})$ based on constant monitoring using the time domain reflectometry method (TRIME-FM moisture meter; IMKO GmbH, Ettlingen, Germany) at a soil depth of 0$30 \mathrm{~cm}$. No rainfall occurred during this period at the study site. Out of 3,200 plants from the four $\mathrm{BC}_{2} \mathrm{~F}_{2}$ populations, total of 135 plants survived under the severe drought stress, and then were harvested individually at the maturing stage. All selected $\mathrm{BC}_{2} \mathrm{~F}_{3}$ lines were progeny tested under the similar drought stress (DS) conditions in the summer of 2009, and $72 \mathrm{BC}_{2} \mathrm{~F}_{3}$ lines with significantly higher yield were selected, ranging from 10 lines from the Jigeng88/MR167 population to 24 lines from the Jigeng88/SN265 population (Table 1 and Figures 1B,C). The flowchart shows the population development for identification of QTL in selected population and validation of QTL in random population (Figure 2).

\section{Phenotypic Data Collection}

In the summers of 2012 and 2013, all selected $72 \mathrm{BC}_{2} \mathrm{~F}_{4}$ lines plus 235 random $\mathrm{BC}_{2} \mathrm{~F}_{4}$ lines from the same four populations were evaluated under the drought stress and irrigated conditions in the experimental farms of NAAFS and Chinese Academy of Agricultural Sciences (CAAS) in Beijing (BJ). The soil of the test field was a sandy loam in Beijing. In each experiment, 30day old seedlings of each ILs were transplanted into a fiverow plot with 30 plants in each plot and a spacing of 20 $\times 25 \mathrm{~cm}$ between plants and rows. A completely randomized block design was used with two replications per line. Under the irrigated control condition, water was applied whenever necessary until most lines had reached the grain-filling stage (total water applied $4,800 \mathrm{~m}^{3} \mathrm{ha}^{-1}$ ). For drought stress, moderate stress condition was performed to validate the DT lines at the NAAFS. A water sheltered facility was adopted in CAAS for creating drought stress. Normal irrigation was maintained for 1 month after transplanting, then the plots were drained and irrigation was withheld completely till harvest. Thus, all tested lines were subjected to severe drought at the reproductive stage. During the periods of drought stress, water levels of the fields were monitored daily at a soil depth of $0-30 \mathrm{~cm}$ based on constant monitoring using the time domain reflectometry method (TRIME-FM moisture meter; IMKO GmbH, Ettlingen, Germany). At maturity, all plants were harvested and measured for grain yield and five plants in each plot were sampled and measured for following yield related traits: heading date (HD), plant height $(\mathrm{PH})$, effective panicle number per plant $(\mathrm{PN})$, 


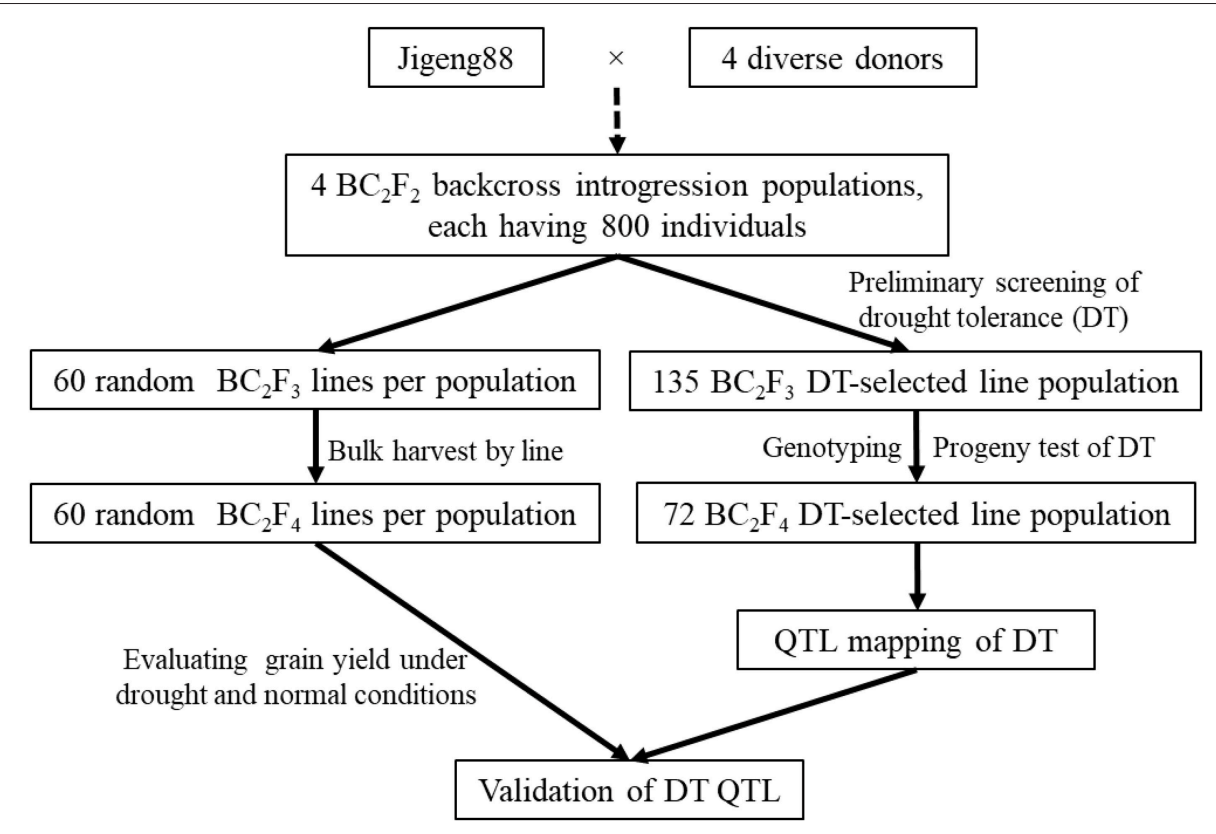

FIGURE 2 | Flowchart of the population development for identification and validation of QTL for drought tolerance in rice.

thousand-grain weight (GW), filled grain number per panicle (GN), grain weight per plant (GY), and spikelet number per panicle (SN). HD was recorded when the panicle was exerted $\sim 50 \%$ of the plants in a plot.

\section{Genotyping Experiments}

Genomic DNA was extracted using the CTAB method (Ahmadikhah, 2009). The DNA was isolated from bulk fresh leaf tissues of each $\mathrm{BC}_{2} \mathrm{~F}_{2: 3} \mathrm{IL}$. More than 600 rice anchor simple sequence repeat (SSR) markers were used to survey the parental lines, resulting in 181,201, 197, and 38 polymorphic SSR markers, respectively, for the four populations. The markers were used to genotype the selected ILs and random ILs.

\section{Statistical Analysis}

Because of the greatly reduced variation among lines within each of the selected IL population, normal statistical methods for identifying QTL in random segregating populations are not appropriate for detecting QTL in selected populations. Therefore, we took a segregation distortion approach to map segregation distortion markers (Cui et al., 2015). In addition, we combined the four selected breeding populations together to perform a joint QTL mapping following the method developed by Cui et al. (2015). However, the four selected populations in this study have different markers. Some markers in some populations were not genotyped. We first developed a consensus map using the multipoint method under the Markov model (Cui et al., 2015). As Cui et al developed, an individual survived from the drought stress with an underlying quantitative trait $y_{j}$ called liability which can be described by the liner model

$$
y_{j}=Z_{j} a+\xi_{j}
$$

where $Z_{j}$ is the genotype indicator for individual $j, a$ is the genetic effect of locus, $\xi_{j}$ is the residual error follow the normal distribution $\xi_{j} \sim N(0,1)$. Assume all the individuals survived are selected based on the $y_{j}>0$ criterion. The probability of surviving is $\operatorname{Pr}\left(y_{j}>0\right)=\Phi\left(Z_{j} a\right)$. Using the Bayes' theorem, the posterior probability of survival for each genotype are $\pi_{j(11)}=$ $\phi_{11} \Phi(a) / \bar{\pi}_{j}, \pi_{j(12)}=\phi_{12} \Phi(0) / \bar{\pi}_{j}, \pi_{j(22)}=\phi_{22} \Phi(-a) / \bar{\pi}_{j}$, where $\bar{\pi}_{j}=\phi_{11} \Phi(a)+\phi_{12} \Phi(0)+\phi_{22} \Phi(-a)$ and $\phi_{11}=13 / 16$, $\phi_{12}=2 / 16, \phi_{22}=1 / 16$ are the expected Mendelian frequencies for the three genotypes in $\mathrm{BC}_{2} \mathrm{~F}_{2}$. When $a=0$, the posterior probabilities are equal to the expected Mendelian frequencies and we will not be able to detect segregation distortion. If $a \neq 0$ the posterior probabilities of genotypes will deviate from the expected Mendelian Segregation ratios. The segregation distortion loci could be identified. For each population, we estimated the effect of each marker and calculated the variance of the estimated effect. Let $\hat{a}_{k}$ be the estimated effect and $\operatorname{var}\left(\hat{a}_{k}\right)$ be the variance of the estimate for a marker under consideration. The Wald test for $H_{0}: a_{k}=0$ in the $k$ th population was obtained using $\hat{a}_{k}^{2} / \operatorname{var}\left(\hat{a}_{k}\right)$. The joint test for all populations for $H_{0}: a_{1}=$ $a_{2}=\ldots=a_{p}=0$ is

$$
\text { Wald }=\sum_{k=1}^{p} \frac{\hat{a}_{k}^{2}}{\operatorname{var}\left(\hat{a}_{k}\right)}
$$

where $p=4$ is the number of populations. When there are multiple populations, the Wald test simply takes the sum of the Wald test of each individual population. In fact, the random model approach was developed by treating each $a_{k}$ as a normally distributed random variable with a common variance across populations, i.e., $a_{k} \sim N\left(0, \sigma_{a}^{2}\right)$ for $k=1, \ldots, p$. The shared variance 
justified the joint mapping (Cui et al., 2015). The critical value for genome-wide significance at the 0.05 level was drawn from 1,000 permuted samples (Churchill and Doerge, 1994).

\section{Construction of Genetic Networks Underlying Drought Tolerance}

According to the molecular quantitative genetic theory (Zhang et al., 2011; Wang et al., 2015), segregating loci at different levels of signaling pathways contributing to DT in the selected ILs from each BC population were expected to show significant frequency shifts and non-random associations in a strictly hierarchical manner. Here, a functional genetic unit (FGU) is defined either as a single locus of significant excess introgression or an associate group (AG) of $r(r \geq 2)$ unlinked but perfectly associated loci of equal introgression in the DT ILs selected from each BC population. Single FGUs of excessive introgression could be detected by the segregation distortion method described above, or by simple $X^{2}$ tests for detecting significant deviations of the donor and genotypic frequencies at individual loci across the genome from the expected allelic and genotypic frequencies estimated from all genotyped markers of the random populations. In addition, DT loci involved in epistasis (the same signaling pathways) were expected to show strong non-random associations in response to selection, and thus could be detected as association groups (AGs) each consisting of $r(r \geq 2)$ unlinked but perfectly associated loci of equal introgression in DT ILs selected from each $\mathrm{BC}$ population. Thus, a multi-locus probability test:

$$
P_{(A G)}=\left(p_{i}\right)^{r m} \times\left(1-p_{i}\right)^{r(n-m)}
$$

where $p_{i}$ is the frequency of the donor introgression in the random ILs from each BC population, $n$ is the number of the selected ILs, mis the number of ILs that have co-introgression of the donor alleles, and $(n-m)$ is the number of ILs having no introgression at the $r$ unlinked loci in the AG. Here $\left(p_{i}\right)^{m}$ is the probability of $m$ ILs having co-introgression of the donor alleles and $\left(1-p_{i}\right)^{n-m}$ is the probability of $(n-m)$ ILs having no introgression at $r$ unlinked loci. The threshold to claim a significant case was $P \leq 0.005$ (Zhang et al., 2014). For each AG consisting of $r(r \geq 2)$ unlinked but perfectly associated loci, $r \times(r-1) / 2$ significant pairwise associations would be existed between the rloci, which were also confirmed by the linkage disequilibrium (LD) analyses. To reveal the multi-locus structure or putative genetic network underlying DT in the ILs from each BC population, pairwise gametic $\mathrm{LD}$ analyses were performed to characterize the relationship between alleles at all DT FGUs detected in the DT ILs from each BC population. The equation of LD statistic

$$
\tilde{D}_{A B}=\tilde{p}_{A B}-\tilde{p}_{A} \tilde{p}_{B}
$$

where $\tilde{p}_{A B}, \tilde{p}_{A}$, and $\tilde{p}_{B}$ were the frequencies of co-introgression functional $\mathrm{AB}$ and functional genotypes at FGUs $\mathrm{A}$ and FGUs $B$, respectively. A multi-locus genetic network including all detected FGUs in the confirmed drought-tolerance ILs was constructed in two steps based on the principle hierarchy: (1) All the FGUs detected in the DT ILs from a single population were divided into major groups according to the LD results. The individual FGUs of different IF within each group were all significantly and positively associated with $\widehat{D} s_{A B}{ }^{\prime}=1.0$, and FGUs in different groups were either independent or negatively associated; (2) Based on the principle of hierarchy, all associated FGUs within each group were connected and formed multiple layers according to their progressively reduced functional genotypes (FG) frequencies and inclusive relationships (Zhang et al., 2011). The underlying assumption for the network construction is that all FGUs in a network are genetically independent (unlinked) from one another, which was true in our cases because all redundant loci due to linkage (recombination frequency $\leq 0.4$ ) associated with each of the FGUs detected in DT ILs of each population were removed.

\section{RESULTS}

\section{Developing ILs With Significantly Improved DT and Yields}

From a total of 3,200 plants in the four $\mathrm{BC}_{2} \mathrm{~F}_{2}$ populations, 135 plants survived the drought stress and were selected in the first round screening. The number of selected individuals ranged from 28 in population I to 40 in population II (Table 1). After progeny testing of the $135 \mathrm{DT} \mathrm{BC}_{2} \mathrm{~F}_{3}$ lines, 17, 21, 10, and 24 DT ILs from the four populations showed significantly higher GY than the recurrent parent (JG88) under drought stress at the reproductive stage. In the phenotyping experiment, all $\mathrm{BC}_{2} \mathrm{~F}_{4}$ IL populations showed significantly improved GY under drought stress when compared to JG88, at Ningxia and/or Beijing (Table 2). The ANOVA results show that there was a statistically significant difference in all traits between the normal and drought stress conditions. The location factor for trait $\mathrm{PH}, \mathrm{PN}, \mathrm{SN}$, GW, and HD were found to be statistically significant with $P$ $<0.01$. There was a significantly difference for PH, PN, GW, and $\mathrm{HD}$ between the different lines. The $\mathrm{G}$ by $\mathrm{E}$ interactions were existed in the trait PH and PN (Supplementary Table 1). The mean yield advantage of the IL populations over the RP ranged from 12.7 to $22.5 \%$ under the moderate drought stress (JG88 suffered 58.5\% yield loss) in Ningxia, and from 89.4 to $178.9 \%$ under the severe drought (JG88 suffered $91.0 \%$ yield loss) in Beijing, even though there was some residual variation for DT among ILs from each population (Table 2). Clearly, the more severe of drought stress was, the greater the yield advantage over the RP the selected DT ILs had. When the yield related traits of the IL populations were examined, almost all populations had similar mean trait values as JG88 except for population I which had significantly higher trait values for $\mathrm{PH}, \mathrm{SN}$, and GN. Under the irrigated conditions, no significant differences were detected for yield and related traits between the selected IL populations and JG88 (Table 2). Nevertheless, we were able to identify five promising ILs which had significant higher yields than JG88 under both drought 
TABLE 2 | Mean performances of the DT introgression lines for grain yield and related traits selected from 4 backcross populations and their recurrent parent (JG88) in Ningxia (2013) and Beijing (2012) under the drought stress and non-stress irrigated conditions.

\begin{tabular}{|c|c|c|c|c|c|c|c|c|c|c|}
\hline & Population & Location & $\mathbf{N}$ & GY(g) & $H D(d)$ & $\mathrm{PH}(\mathrm{cm})$ & PN & GN & SN & GW(g) \\
\hline \multirow[t]{10}{*}{ Drought stress } & 1 & $N X$ & 17 & $8.4 \pm 1.8^{*}$ & $108.1 \pm 6.1$ & $84.5 \pm 13.3^{*}$ & $4.7 \pm 0.6$ & $121.3 \pm 17.0^{\star \star}$ & $151.1 \pm 20.0^{\star \star}$ & $17.8 \pm 1.5$ \\
\hline & $\|$ & $N X$ & 21 & $8.1 \pm 1.0^{*}$ & $101.6 \pm 3.7$ & $67.5 \pm 6.7$ & $5.3 \pm 0.7^{*}$ & $94.0 \pm 14.8$ & $126.5 \pm 18.9$ & $18.0 \pm 1.2$ \\
\hline & III & NX & 10 & $8.7 \pm 1.5^{\star \star}$ & $103.3 \pm 4.1$ & $69.8 \pm 4.8$ & $5.7 \pm 0.8^{\star \star}$ & $93.3 \pm 16.1$ & $119.4 \pm 15.9$ & $19.0 \pm 1.7$ \\
\hline & IV & NX & 24 & $8.0 \pm 1.2^{\star}$ & $106.4 \pm 1.4$ & $74.2 \pm 3.3$ & $5.2 \pm 0.6$ & $97.4 \pm 10.2$ & $111.6 \pm 10.6$ & $18.8 \pm 1.8$ \\
\hline & JG88(RP) & NX & & $7.1 \pm 0.4$ & $105.1 \pm 1.9$ & $73.6 \pm 2.1$ & $4.7 \pm 0.4$ & $87.9 \pm 3.3$ & $113.4 \pm 15.1$ & $20.3 \pm 2.4$ \\
\hline & I & BJ & 17 & $4.5 \pm 2.1$ & $96.9 \pm 8.8^{\star}$ & $89.1 \pm 12.4^{*}$ & $2.1 \pm 1.2$ & $114.8 \pm 46.7$ & $150.4 \pm 50.9$ & $14.9 \pm 1.9$ \\
\hline & $\|$ & BJ & 21 & $5.3 \pm 1.8$ & $93.3 \pm 3.9$ & $79.0 \pm 7.1$ & $3.0 \pm 1.4$ & $119.0 \pm 54.8$ & $170.9 \pm 123.4$ & $16.7 \pm 1.4$ \\
\hline & III & BJ & 10 & $4.6 \pm 2.5$ & $91.7 \pm 3.3$ & $81.2 \pm 5.6$ & $2.2 \pm 1.4$ & $130.1 \pm 60.7^{*}$ & $155.4 \pm 62.9$ & $16.1 \pm 2.4$ \\
\hline & IV & BJ & 24 & $3.6 \pm 1.6$ & $98.0 \pm 3.0^{\star \star}$ & $89.1 \pm 5.4^{\star}$ & $1.9 \pm 0.9$ & $111.2 \pm 52.8$ & $141.0 \pm 66.6$ & $16.1 \pm 1.9$ \\
\hline & JG88(RP) & BJ & & $1.9 \pm 0.5$ & $92.5 \pm 3.2$ & $78.5 \pm 6.6$ & $2.2 \pm 1.0$ & $97.7 \pm 56.2$ & $138.7 \pm 60.2$ & $15.7 \pm 1.9$ \\
\hline \multirow{10}{*}{$\begin{array}{l}\text { Non-stress irrigated } \\
\text { conditions }\end{array}$} & 1 & NX & 17 & $17.4 \pm 3.9$ & $102.5 \pm 5.0$ & $95.8 \pm 10.7$ & $5.9 \pm 1.0$ & $184.1 \pm 22.0$ & $204.5 \pm 21.7$ & $20.3 \pm 1.3$ \\
\hline & $\|$ & NX & 21 & $18.5 \pm 2.7$ & $96.9 \pm 2.3$ & $93.3 \pm 7.4$ & $6.2 \pm 1.2$ & $175.7 \pm 26.2$ & $192.5 \pm 27.7$ & $21.5 \pm 1.4$ \\
\hline & III & $N X$ & 10 & $16.9 \pm 3.8$ & $99.4 \pm 3.9$ & $87.4 \pm 6.4$ & $5.9 \pm 0.9$ & $157.7 \pm 30.5$ & $169.1 \pm 31.8$ & $22.3 \pm 1.9$ \\
\hline & IV & NX & 24 & $18.9 \pm 3.1$ & $101.6 \pm 1.8$ & $99.4 \pm 3.4$ & $6.3 \pm 0.9$ & $175.7 \pm 20.0$ & $187.3 \pm 19.7$ & $21.3 \pm 1.2$ \\
\hline & JG88(RP) & NX & & $17.1 \pm 1.4$ & $99.0 \pm 2.6$ & $91.0 \pm 4.8$ & $6.0 \pm 0.6$ & $175.1 \pm 28.7$ & $186.6 \pm 31.8$ & $21.5 \pm 2.1$ \\
\hline & 1 & BJ & 17 & $22.6 \pm 4.5$ & $93.3 \pm 6.8$ & $114.2 \pm 14.1^{\star \star}$ & $7.3 \pm 1.1$ & $217.3 \pm 31.9$ & $245.8 \pm 29.0$ & $19.1 \pm 1.2$ \\
\hline & $\|$ & BJ & 21 & $20.0 \pm 2.4$ & $90.9 \pm 5.1$ & $104.4 \pm 6.1$ & $6.2 \pm 0.8$ & $206.4 \pm 37.9$ & $237.2 \pm 44.9$ & $19.6 \pm 1.2$ \\
\hline & III & BJ & 10 & $21.6 \pm 6.3$ & $91.5 \pm 4.8$ & $100.2 \pm 7.1$ & $6.8 \pm 1.2$ & $192.6 \pm 32.6$ & $208.2 \pm 31.5$ & $20.6 \pm 1.0$ \\
\hline & IV & BJ & 24 & $22.1 \pm 2.4$ & $96.2 \pm 1.7$ & $109.8 \pm 3.6$ & $6.7 \pm 0.8$ & $214.4 \pm 19.3$ & $226.2 \pm 20.3$ & $20.0 \pm 1.3$ \\
\hline & JG88(RP) & BJ & & $21.1 \pm 4.7$ & $94.9 \pm 4.5$ & $106.5 \pm 2.1$ & $7.4 \pm 1.7$ & $222.0 \pm 25.8$ & $241.0 \pm 29.3$ & $19.6 \pm 2.2$ \\
\hline
\end{tabular}

GY, grain yield; PH, plant height; PN, effective panicle number per plant; GN, filled grain number per panicle; SN, spike number per panicle; GW, thousand grain weight; HD, head date. "and ${ }^{* *}$ indicate the significance levels of $P=0.05$ and 0.01 , respectively, based on Duncan's multiple comparisons in ANOVA.

stress and normal irrigated conditions in both and/or either environments (Table 3).

\section{Detection and Validation of DT QTL in the Selected and Random IL Populations}

Using a critical value of Wald test of 16.97 drawn from 1,000 permutated samples, 13 QTLs on seven rice chromosomes were detected in 72 ILs selected from the four BC populations (Table 4). These included 7 QTL (QDT1.3, QDT2.4, QDT2.9, QDT7.1, QDT7.2, QDT7.4, and QDT11.5) in the 17 ILs from population JG88/IR66897B(I), 7 QTL (QDT1.4, QDT6.3, QDT2.9, QDT6.3, QDT6.5, and QDT10.3) from 21 ILs from population JG88/MR77(II), 3 QTL (QDT2.9, QDT6.3, and QDT6.5) from 10 ILs from population JG88/MR167 (III), and 4 QTL (QDT1.3, QDT2.4, QDT6.3, and QDT6.5) from the 24 ILs of JG88/SN265(IV). Of these, 2 QTL (QDT2.9 and QDT6.3) each was detected in three of the populations, 4 QTL (QDT1.3, QDT1.4, QDT6.5, and QDT7.1) each was detected in two of the populations, and the 7 remaining QTL each was detected in a single population. Two QTL, QDT1.4 near marker RM449 on chromosome 1 and QDT2.9 near MR266 on chromosome 2 were identified with the highest Wald values of 65.8 and 41.54 .

In order to validate the detected DT QTL, the four random (unselected) populations each with $\sim 60 \mathrm{BC}_{2} \mathrm{~F}_{4}$ lines from the same four crosses were evaluated under drought stress in the replicated trials. Based on the genotypic differences in mean yields under drought at each of the detected QTL in the random $\mathrm{BC}_{2} \mathrm{~F}_{4}$ lines from each population, 11 of the 13 detected QTL could be validated in the random populations evaluated in the Ningxia experiment and 8 of the DT QTL could be validated in the random populations in the Beijing experiment (Table 5). In all cases, significantly increased GY under drought stress in both Ningxia and Beijing were associated with the donor homozygote genotype, indicating that the donor alleles at all these detected QTL were associated with DT. The average yield advantage under drought from the donor homozygote at individual QTL ranged from $4.1 \%$ for QDT8.3 to $45.9 \%$ for QDT10.3.

\section{Putative Genetic Networks (Multi-Locus Structures) Underlying DT}

Table 6 shows 29 FGUs (28 single loci and 1 association group or AG) for DT detected by $\chi^{2}$ tests (single loci) and multilocus linkage disequilibrium analyses in 72 drought-tolerant introgression lines (ILs) selected from the four populations. These included 10 FGUs detected in 17 ILs of JG88/IR66897B (I), 9 loci in eight FGUs in 21 ILs of JG88/MR77 (II), 4 FGUs in 10 ILs of JG88/MR167 (III), and 7 FGUs in 24 ILs of JG88/SN265(IV), respectively (Table 6 and Figure 3). The average introgression frequency (IF) of the donor alleles at the $30 \mathrm{DT}$ loci ranged from 0.147 to 0.81 .

Figure 3 shows the four putative genetic networks or multilocus structures each containing all DT FGUs identified in 


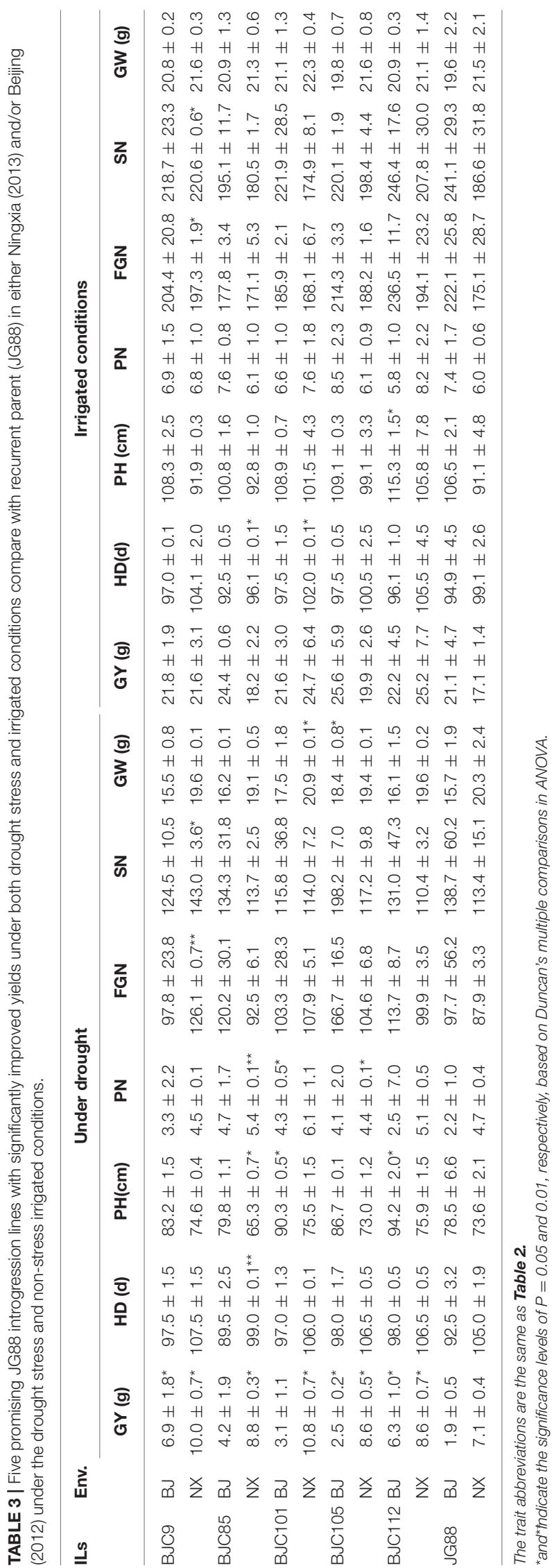

the selected DT ILs from populations I-IV. Genetic network I contains 10 FGUs (10 loci) in five largely independent branches detected in the 17 DT ILs from population Jigeng88/IR66897B. Branch I-1 consisted of two unlinked but perfectly associated loci, with RM542 (bin 7.4) of high introgression placed in the upstream (IF $=0.588$ ) and RM480 (bin 5.8) of lower introgression ( $\mathrm{IF}=0.294$ ) in the downstream. Similarly, branches I-2, I-3, and I-4 each also consisted of two unlinked but perfectly associated loci with a locus of high introgression placed in the upstream and a locus of low introgression placed in the downstream. Branch I-5 contains a single locus near RM141 (bin 6.7). Genetic network II consisted of eight FGUs (9 loci) with two major branches plus two independent loci detected in the 21 DT ILs from population Jigeng88/MR77. Branch II-1 had RM449 (Bin1.1) of very high introgression $(\mathrm{IF}=0.810)$ in the upstream as the putative regulator connected with three sub-branches, RM286 (bin11.1) $(\mathrm{IF}=0.333), \mathrm{RM} 470$ (bin 4.6) $(\mathrm{IF}=0.214)$, and $a g D T_{I I-1}$ consisting of two unlinked but perfectly associated loci at RM336 (Bin7.6) and RM331 (Bin8.3) of low introgression (IF $=0.286)$ in the downstream. Branch II-2 consisted of 3 unlinked but highly associated FGUs with RM406 of high introgression placed at the top as the putative regulator and 2 FGUs (RM276 in Bin6.3 and RM311 in Bin10.3) of lower introgression in the downstream. The independent FGUs included RM541 at bin 6.5 of high introgression (IF $=0.429)$ which were detected as DT QTL using the segregation distortion method (Table 3). Genetic network III detected in the 10 ILs of JG88/MR167 consisted of 4 FGUs (4 loci) with a single branch plus two independent loci (MR518 in bin4.2 and MR253 in bin6.3). Branch III-1 containing 2 unlinked but highly associated loci with MR339 (bin8.3) in the upstream and MR406 (bin2.9) in the downstream. The genetic network of JG88/SN265 contained only 7 independent loci (FGUs). This was not surprising since both JG88 and SN265 are closely related Geng varieties from Northeast China.

\section{DISCUSSION}

In this study, we have shown that most selected ILs from the four BC populations had significantly improved GY under drought stress without yield penalty under the normal irrigated conditions (Table 2). This result plus the development of five promising ILs that had significantly higher yields under both drought stress and normal irrigated conditions indicated that backcross breeding was effective for improving DT of high yielding Geng varieties. We noted that the improved yield performances of ILs under drought stress were associated primarily with increased PN and GN, but not with increased TGW. This is consistent with the effect of natural selection which tends to act on the number instead of size of rice grains (Ashikari et al., 2005; Lu et al., 2013). It should be pointed out that none of the four donors in our BC breeding has good DT, but they apparently all have genes contributing to DT, indicating the presence of rich "hidden" genetic diversity in the primary gene pool of rice for DT, as reported previously (Lafitte et al., 2006; He et al., 2010; Wang et al., 2013a,b; Ali et al., 2017). This is true for tolerances to other abiotic stresses such as salinity, submergence, 


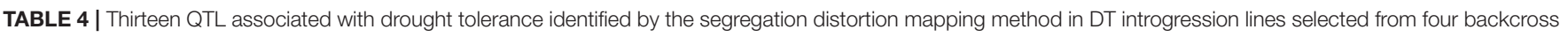
populations.

\begin{tabular}{|c|c|c|c|c|c|}
\hline QTL & Marker & Position (cM) ${ }^{a}$ & Wald value $^{b}$ & Populationc & QTL and drought-responsive genes \\
\hline QDT1.3 & RM572 & 66.4 & 22.15 & I, II & rfw1b (Li et al., 2005a) \\
\hline QDT1.4 & RM449 & 78.4 & 65.8 & II, IV & brt1d (Li et al., 2005a) \\
\hline QDT2.4 & RM424 & 66 & 17.5 & I & qDTY2.1 (Dixit et al., 2012); qLRS-2 (You et al., 2006) \\
\hline QDT2.8 & RM425 & 166 & 32.72 & $\|$ & \\
\hline QDT2.9 & RM266 & 192.2 & 41.54 & I, II, III & OsPIP1;3 (Lian et al., 2004; Liu et al., 2007) \\
\hline QDT6.3 & RM276 & 40.3 & 37.87 & II, III, IV & \\
\hline QDT6.5 & RM541 & 75.5 & 27.82 & II, IV & qgy6.1 (Palanog et al., 2014) \\
\hline QDT7.1 & RM427 & 1.1 & 20.74 & I, IV & OsCIPK23 (Yang et al., 2008) \\
\hline QDT7.2 & RM125 & 24.8 & 25.12 & I & OsNAC3/ONAC067 (Ooka et al., 2003) \\
\hline QDT7.4 & RM542 & 34.7 & 30.42 & I & rn7a (Li et al., 2005a) \\
\hline QDT8.3 & RM339 & 72.2 & 22.05 & III & QGy8 (Xu et al., 2005); QPn8, QTgw8, QSf8 (Wang et al., 2012) \\
\hline QDT10.3 & RM311 & 25.2 & 19.82 & $\|$ & trdw10.1 (Nguyen et al., 2004) \\
\hline QDT11.5 & RM229 & 77.8 & 21.75 & । & \\
\hline
\end{tabular}

${ }^{a} \mathrm{CM}$ means centimorgan, a unit of genetic distance.

${ }^{b}$ Wald value $=16.97$ and 20.35 at $P=0.05$ and 0.01 , respectively.

${ }^{c}$ The designations of the populations are the same as Table 1.

TABLE 5 | Phenotype validation of DT QTLs by comparing mean grain yields of QTL genotypes of the four random populations in Ningxia (2013) and Beijing (2012) under the drought condition.

\begin{tabular}{|c|c|c|c|c|c|c|c|c|c|}
\hline QTL & Marker & Population & \multicolumn{3}{|c|}{ Mean grain yield (g/plant) in Ningxia } & \multicolumn{3}{|c|}{ Mean grain yield ( $g /$ plant) in Beijing } & Yield improvement ${ }^{\mathrm{a}}(\%)$ \\
\hline QDT2.4 & RM424 & 1 & 10.30 & 8.8 & 7.38 & & & & 39.6 \\
\hline QDT2.8 & RM425 & $\|$ & 10.25 & 8.46 & 7.63 & 8.38 & 6.87 & 6.52 & 31.4 \\
\hline QDT2.9 & RM266 & I & 10.23 & 9.05 & 7.38 & 6.39 & 5.91 & 5.41 & 28.4 \\
\hline \multirow[t]{2}{*}{ QDT6.5 } & RM541 & $\|$ & 10.10 & 8.58 & 7.63 & 8.60 & 6.81 & 6.42 & 24.2 \\
\hline & & IV & & & & 8.20 & 6.70 & 6.89 & 19.0 \\
\hline \multirow[t]{2}{*}{ QDT7.1 } & RM427 & I & 11.56 & 8.72 & 8.82 & 6.86 & 6.10 & 5.41 & 28.9 \\
\hline & & IV & 9.03 & 8.31 & 7.71 & & & & 17.1 \\
\hline QDT7.4 & RM542 & 1 & 9.52 & 9.10 & 8.82 & & & & 8.0 \\
\hline
\end{tabular}

a Yield improvement (\%) $=($ mean GY of ILs carrying the donor allele - mean GY of JG88)/mean GY of JG88.

high and low temperatures, etc. (Ali et al., 2006; Cheng et al., 2011; Zhang et al., 2014) as well as for almost all complex traits in rice (Li and Rutger, 2000; Zhang, 2012).

Rice responses to drought and other abiotic stresses are known to be controlled by complex gene networks consisting of many signaling pathways (Wang et al., 2011, 2015). However, it remains a great challenge to link results from the classic QTL mapping with those of molecular and transcriptomic analyses. Resembling the previously reported genetic networks underlying rice tolerances to cold and submergence (Zhang et al., 2014; Wang et al., 2015), the genetic networks underlying DT detected in this study each consisted of multiple independent branches, each might represent a signaling pathway involved in rice DT based on the following pieces of evidence. First, all DT QTL of large effects (high introgression) detected in this study (Table 4) appeared to be real ones as they were all validated in the random $\mathrm{BC}$ populations of small population sizes that are known to be less powerful in detecting QTL. Secondly, seven of the major DT QTL (QDT1.3, QDT1.4, QDT2.4, QDT6.5, QDT7.4, QDT8.3, and QDT11.5) had high introgression and were placed as putative regulators in the upstream positions of the genetic networks (Figure 3), as predicted by the theory (Zhang et al., 2011). Two other putative regulators at bins 1.3 (branch I-4) and 2.8 (branch II-2) were also in the approximate vicinity 
TABLE 6 | Genomic information for 29 functional genetic units (FGUs) (28 single loci and 1 association groups or AGs) for drought tolerance (DT) detected by $\chi^{2}$ tests (single loci) and multi-locus linkage disequilibrium analyses in 72 drought-tolerant introgression lines (ILs) selected from four populations.

\begin{tabular}{|c|c|c|c|c|c|c|c|c|c|c|}
\hline Donor & Code & $\mathrm{AG}^{\mathrm{a}}$ & Branch & Marker & $\mathrm{Bin}^{\mathrm{b}}$ & Position (cM)c & $B^{d}$ & $\mathbf{H}$ & IF & $P$-value \\
\hline IR66897B & 1 & & $\mid-1$ & RM542 & 7.4 & 34.7 & 10 & 0 & 0.588 & $1.40 \mathrm{E}-11$ \\
\hline IR66897B & I & & $\mid-1$ & RM480 & 5.8 & 130.6 & 5 & 0 & 0.294 & 1.49E-02 \\
\hline IR66897B & 1 & & $\mathrm{I}-2$ & RM229 & 11.5 & 77.8 & 8 & 2 & 0.529 & $2.66 \mathrm{E}-10$ \\
\hline IR66897B & 1 & & $\mathrm{I}-2$ & RM427 & 7.1 & 1.1 & 8 & 0 & 0.471 & 4.70E-07 \\
\hline IR66897B & 1 & & $1-3$ & RM424 & 2.4 & 66 & 7 & 1 & 0.441 & 6.66E-06 \\
\hline IR66897B & 1 & & $1-3$ & RM276 & 6.3 & 40.3 & 4 & 0 & 0.235 & 1.67E-02 \\
\hline IR66897B & 1 & & $\mid-4$ & RM572 & 1.3 & 61.2 & 7 & 0 & 0.412 & 3.38E-05 \\
\hline IR66897B & I & & $\mid-4$ & RM266 & 2.9 & 192.2 & 5 & 0 & 0.294 & 1.67E-02 \\
\hline IR66897B & 1 & & $\mid-4$ & RM167 & 11.2 & 37.5 & 1 & 3 & 0.147 & 6.35E-08 \\
\hline IR66897B & 1 & & & RM141 & 6.7 & 143.7 & 6 & 0 & 0.353 & 9.50E-04 \\
\hline MR77 & $\|$ & & $\|-1$ & RM449 & 1.1 & 78.4 & 17 & 0 & 0.81 & $9.56 \mathrm{E}-29$ \\
\hline MR77 & $\|$ & $a g D T_{\|-1}$ & $\|-1$ & RM336 & 7.6 & 61 & 6 & 0 & 0.286 & 8.36E-03 \\
\hline MR77 & $\|$ & $a g D T_{/ l-1}$ & $\|-1$ & RM331 & 8.3 & 69 & 6 & 0 & 0.286 & 8.36E-03 \\
\hline MR77 & $\|$ & & $\|-1$ & RM286 & 11.1 & 0 & 7 & 0 & 0.333 & 6.32E-04 \\
\hline MR77 & $\|$ & & $\|-1$ & RM470 & 4.6 & 115.5 & 4 & 1 & 0.214 & 1.14E-01 \\
\hline MR77 & $\|$ & & $\|-2$ & RM406 & 2.8 & 186.4 & 12 & 0 & 0.571 & $3.03 E-13$ \\
\hline MR77 & $\|$ & & $\|-2$ & RM276 & 6.3 & 40.3 & 9 & 1 & 0.452 & 9.35E-06 \\
\hline MR77 & $\|$ & & $\|-2$ & RM311 & 10.3 & 25.2 & 9 & 1 & 0.452 & $2.14 \mathrm{E}-07$ \\
\hline MR77 & $\|$ & & & RM541 & 6.5 & 75.5 & 9 & 0 & 0.429 & $6.53 \mathrm{E}-07$ \\
\hline MR167 & III & & || $\mid-1$ & RM339 & 8.3 & 72.2 & 6 & 0 & 0.6 & 2.04E-07 \\
\hline MR167 & III & & & RM406 & 2.9 & 186.4 & 4 & 0 & 0.4 & 3.39E-03 \\
\hline MR167 & III & & & RM518 & 4.2 & 25.5 & 4 & 0 & 0.4 & 3.39E-03 \\
\hline MR167 & III & & & RM253 & 6.3 & 37 & 4 & 0 & 0.4 & 3.39E-03 \\
\hline SN265 & IV & & & RM449 & 1.4 & 78.4 & 8 & 3 & 0.396 & 4.95E-08 \\
\hline SN265 & IV & & & RM506 & 8.1 & 0 & 8 & 1 & 0.354 & 1.81E-04 \\
\hline SN265 & IV & & & RM541 & 6.5 & 75.5 & 7 & 2 & 0.333 & 5.77E-05 \\
\hline SN265 & IV & & & RM585 & 6.3 & 25.1 & 7 & 2 & 0.333 & 5.77E-05 \\
\hline SN265 & IV & & & RM426 & 3.9 & 157.3 & 7 & 0 & 0.292 & 4.42E-03 \\
\hline SN265 & IV & & & RM481 & 7.1 & 3.2 & 6 & 0 & 0.25 & 3.28E-02 \\
\hline SN265 & IV & & & $\mathrm{RM} 283$ & 1.2 & 31.4 & 6 & 0 & 0.25 & 3.28E-02 \\
\hline
\end{tabular}

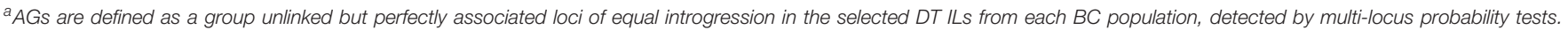
$P$-value is the probabilities for the null hypothesis that the genotypic frequencies fit the Mendelian segregation based on single locus $X^{2}$ tests.

${ }^{b}$ Bold ones were DT QTLs detected by the segregation distortion approach in Table 2.

${ }^{c} \mathrm{CM}$ means centimorgan, a unit of genetic distance.

${ }^{d} \mathrm{~B}, \mathrm{H}$, and IF are the frequencies of the donor homozygote, heterozygote, and donor introgression frequency in the selected DT ILs from each population.

to QDT1.4 and QDT2.9, suggesting they were likely due to linkage. Thirdly, according to the QTLs/genes located in the region within $200 \mathrm{~kb}$ are the same QTL/gene, we found that most of the major QTL identified in this study were mapped to approximately the same locations as previously reported QTL or important regulatory genes for DT (Table 4). For example, QDT7.2 (near RM125) was mapped to the genomic location harboring ONAC067/OsNAC3, a member of plant-specific NAC family that is known to regulate plant responses to drought, cold and high salinity (Kikuchi et al., 2003; Takasaki et al., 2010). This region also harbor two previously reported DT QTL in rice detected in a set of recombinant inbred lines developed from the cross between Zhenshan 97 (Xian) and a upland rice cultivar IRAT109 (tropical Geng; Yue et al., 2005, 2008). Similarly, QDT2.9 was mapped together with OsPIP1-3, a gene that showed increased transcription in response to drought and probably played an important role in drought avoidance in rice (Lian et al., 2004). The QDT7.1 was mapped in the region with a cloned gene OsCIPK23 which is a multi-stress induced gene mediates a signaling pathway commonly shared by both pollination and drought stress. The QDTY2.4 was mapped to the same location as most a previously reported DT QTL, qDTY2.1, and $q L R S-2$ (Dixit et al., 2012). The QDT1.3 and QDT1.3 located on chromosome 1 were mapped in the adjacent region harboring $r f w 1 b$ and brt1d (Li et al., 2005a). The QDT8.3 was mapped in the same region of QGy8 for grain yield, QPn8 for panicle number, QTgw8 for thousand grain weight and QSf8 for seed fertility (Wang et al., 2012) under drought stresses. The QDT10.3 was mapped to the same region as the $\operatorname{trdw10.1}$ was reported by Nguyen et al. (2004). The QDT2.8, QDT6.3, and QDT11.5 are probably new DT QTLs which have been validated in random population of this study. All these results strongly suggested that most main-effect 

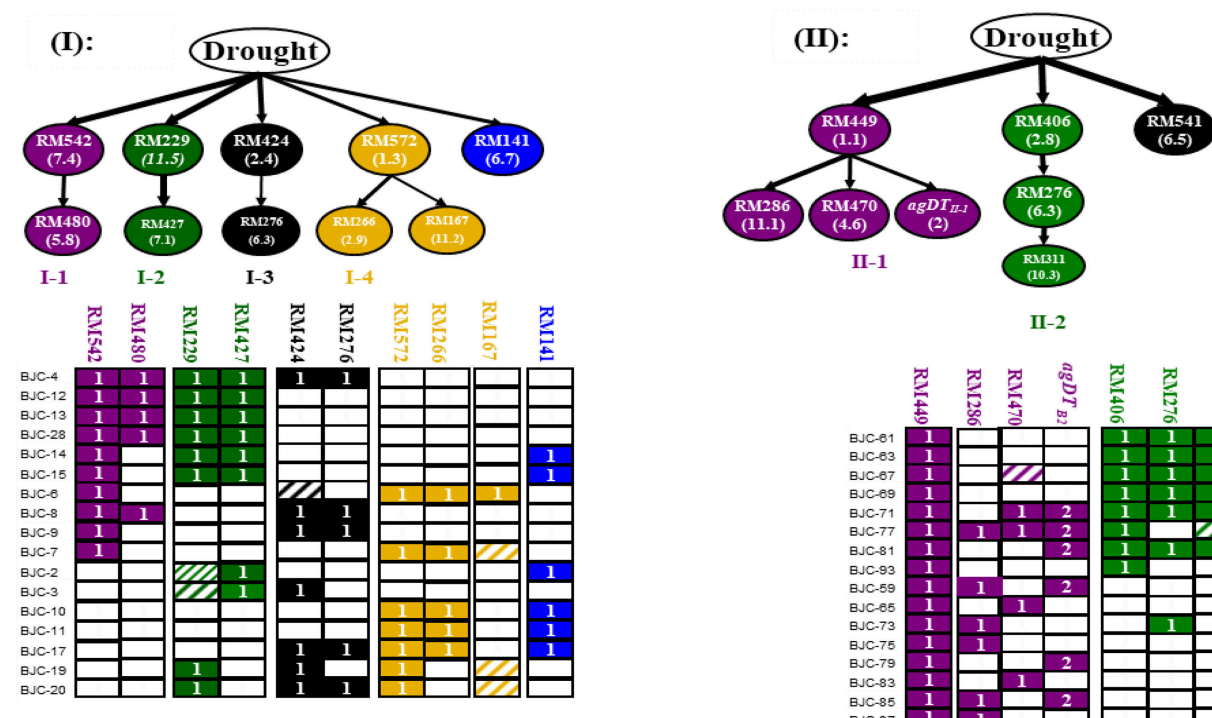

II-2
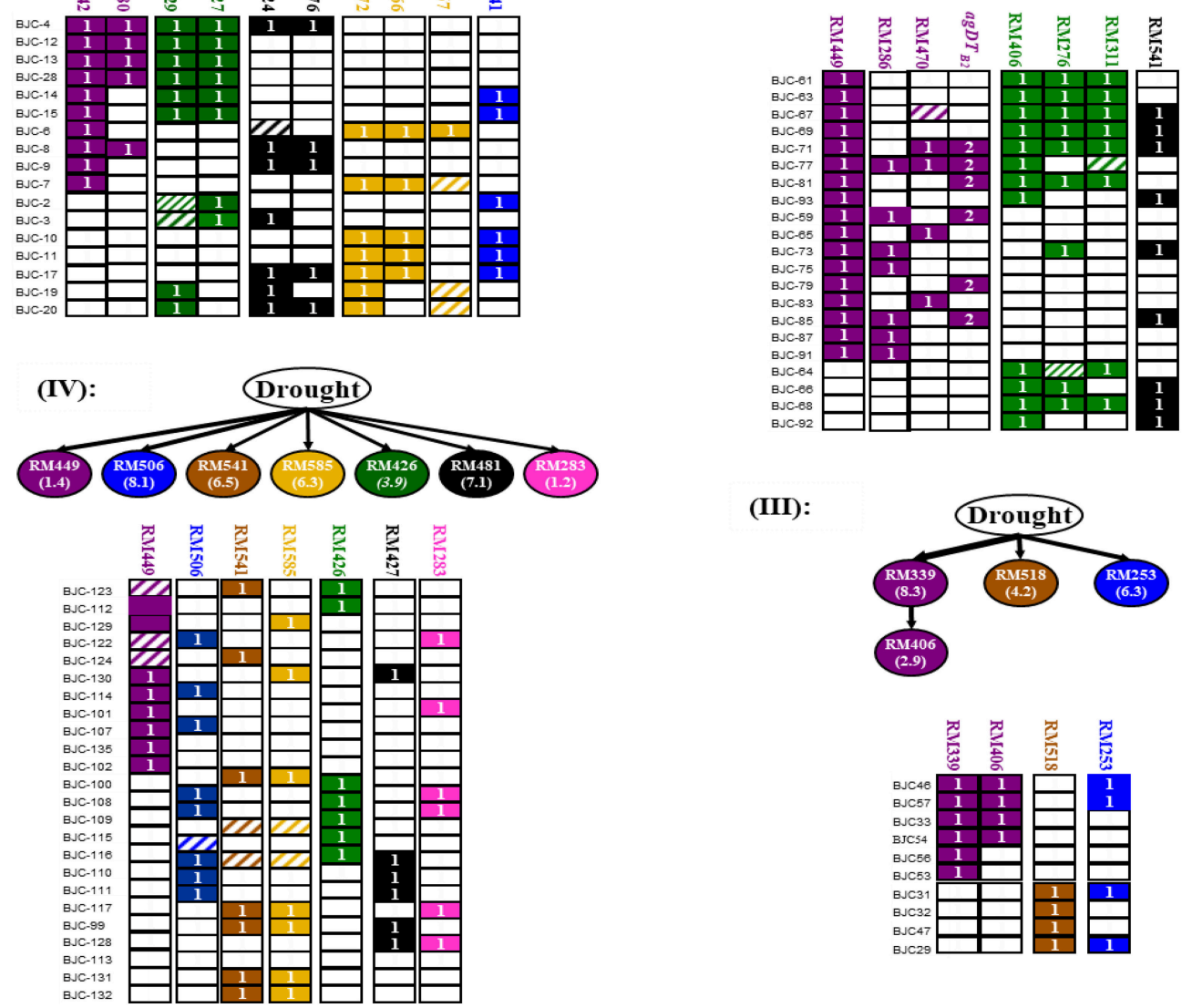

(III):
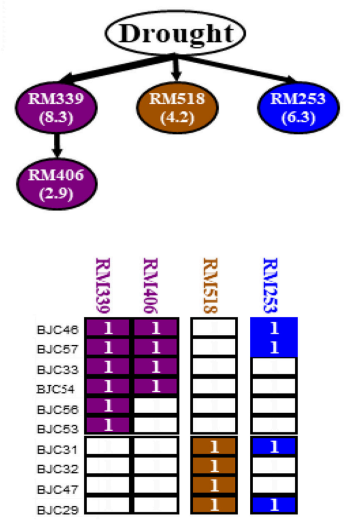

FIGURE 3 | Putative genetic networks (multi-locus structures) underlying drought tolerance (DT) of rice detected in JG88 backcross introgression lines (BILs) from four populations. In the corresponding graphical genotypes of each network, the unfilled, fully colored, and patched cells represent the recipient homozygote, donor homozygote, and heterozygote genotypes. The numbers in the cells of each FGU are the number of loci included in the FGUs. The loci (markers) included in each of the detected association groups (AGs) are shown in Table 6. Solid arrow lines connected two FGUs in each branch of a network represent putative functional relationships with those of high introgression as putative regulators in the upstream and those of low introgression in the downstream, and the thickness of an arrow line was proportional to the introgression frequency of the downstream FGU in Table 6.

QTL detected in this study were most likely regulatory genes that play important roles in regulating rice responses to DT and probably other abiotic stresses. We noted that 8 additional downstream FGUs by our non-random association analyses were undetectable by the segregation distortion method (SDM). This was due partially to the fact that SDM considers only allelic frequency shifts but not on the deviation of genotypic frequency shifts from direction selection, and primarily to its inability to detect epistasis (Cui et al., 2015).

Finally, the DT ILs and the genetic information regarding the DT QTL and the network (Supplementary Table 2) they carry provide useful materials and information for further improving rice DT and yield by designed QTL pyramiding (Zhang et al., 2014; Ali et al., 2017). As we noted in Table 2, there were considerable residual variation among individual ILs for GY and related traits, and so were for different QTL from different donors in their genetic compositions (Figure 1). According to our experiences, it is hoped that better and promising progeny that combine higher levels of DT and grain yield can be readily achieved using this breeding strategy with relatively short period of time and limited breeding effort (Ali et al., 2017). 


\section{AUTHOR CONTRIBUTIONS}

YC analyzed the data and wrote the manuscript; WZ and XL were in charge of the field experiment management; SX developed the statistical model; JX designed and performed the experiment; ZL conceived the study and were in charge of the direction and planning.

\section{ACKNOWLEDGMENTS}

The project was supported by the National Natural Science Foundation of China-Consultative Group on International

\section{REFERENCES}

Ahmadikhah, A. (2009). A rapid mini-prep DNA extraction method in rice (Oryza sativa). Afr. J. Biotechnol. 8, 234-238.

Ali, A. J., Xu, J. L., Ismail, A. M., Fu, B. Y., Vijaykumar, C. H. M., Gao, Y. M., et al. (2006). Hidden diversity for abiotic and biotic stress tolerances in the primary gene pool of rice revealed by a large backcross breeding program. Field Crops Res. 97, 66-76. doi: 10.1016/j.fcr.2005.08.016

Ali, J., Xu, J. L., Gao, Y. M., Ma, X. F., Meng, L. J., Wang, Y., et al. (2017). Harnessing the hidden genetic diversity for improving multiple abiotic stress tolerance in rice (Oryza sativa L.). PLoS ONE 12:e172515. doi: 10.1371/journal.pone.0172515

Ashikari, M., Sakakibara, H., Lin, S., Yamamoto, T., Takashi, T., Nishimura, A., et al. (2005). Cytokinin oxidase regulates rice grain production. Science 309, 741-745. doi: 10.1126/science.1113373

Cheng, L., Wang, Y., Meng, L., Hu, X., Cui, Y., Sun, Y., et al. (2011). Identification of salt-tolerant QTLs with strong genetic background effect using two sets of reciprocal introgression lines in rice. Genome 55, 45-55. doi: 10.1139/g11-075

Churchill, G. A., and Doerge, R. W. (1994). Empirical threshold values for quantitative trait mapping. Genetics 138, 963-971.

Cui, Y., Zhang, F., Xu, J., Li, Z., and Xu, S. (2015). Mapping quantitative trait loci in selected breeding populations: a segregation distortion approach. Heredity 115, 538-546. doi: 10.1038/hdy.2015.56

Dixit, S., Swamy, B. P., Vikram, P., Ahmed, H. U., Sta Cruz, M. T., Amante, M., et al. (2012). Fine mapping of QTLs for rice grain yield under drought reveals sub-QTLs conferring a response to variable drought severities. Theor. Appl. Genet. 125, 155-169. doi: 10.1007/s00122-012-1823-9

Fukai, S., and Cooper, M. (1995). Development of drought-resistant cultivars using physiomorphological traits in rice. Field Crops Res. 40, 67-86. doi: 10.1016/0378-4290(94)00096-U

Guan, Y. S., Serraj, R., Liu, S. H., Xu, J. L., Ali, J., Wang, W. S., et al. (2010). Simultaneously improving yield under drought stress and non-stress conditions: a case study of rice (Oryza sativa L.). J. Exp. Bot. 61, 4145-4156. doi: $10.1093 /$ jxb/erq212

He, Y., Zheng, T., Hao, X., Wang, L., Gao, Y., Hua, Z., et al. (2010). Yield performances of japonica introgression lines selected for drought tolerance in a BC breeding programme. Plant Breed. 129, 167-175. doi: 10.1111/j.1439-0523.2009.01683.x

Ji, K., Wang, Y., Sun, W., Lou, Q., Mei, H., Shen, S., et al. (2012). Droughtresponsive mechanisms in rice genotypes with contrasting drought tolerance during reproductive stage. J. Plant Physiol. 169, 336-344. doi: 10.1016/j.jplph.2011.10.010

Kamoshita, A., Babu, R. C., Boopathi, N. M., and Fukai, S. (2008). Phenotypic and genotypic analysis of drought-resistance traits for development of rice cultivars adapted to rainfed environments. Field Crop Res. 109, 1-23. doi: 10.1016/j.fcr.2008.06.010

Kikuchi, S., Satoh, K., Nagata, T., Kawagashira, N., Doi, K., Kishimoto, N., et al. (2003). Collection, mapping, and annotation of over 28,000 cDNA clones from japonica rice. Science 301, 376-379. doi: 10.1126/science.1081288

Lafitte, H., Li, Z., Vijayakumar, C., Gao, Y., Shi, Y., Xu, J., et al. (2006). Improvement of rice drought tolerance through backcross breeding: evaluation
Agriculture Research (31261140369) to JX, Fundamental Research Funds for Central Non-profit Scientific Institution (Y2016PT53) to JX, The 863 GSR grants (2014AA10A601) to JX and Shenzhen Peacock Plan and BMGF grants (GD1393) to ZL. YC was supported by the Beachell-Borlaug International Student Fellowship from Monsanto.

\section{SUPPLEMENTARY MATERIAL}

The Supplementary Material for this article can be found online at: https://www.frontiersin.org/articles/10.3389/fpls.2018. 00320/full\#supplementary-material of donors and selection in drought nurseries. Field Crop Res. 97, 77-86. doi: 10.1016/j.fcr.2005.08.017

Li, Z., Mu, P., Li, C., Zhang, H., Li, Z., Gao, Y., et al. (2005a). QTL mapping of root traits in a doubled haploid population from a cross between upland and lowland japonica rice in three environments. Theor. Appl. Genet. 110, 1244-1252. doi: 10.1007/s00122-005-1958-Z

Li, Z., and Rutger, J. (2000). Geographic distribution and multilocus organization of isozyme variation of rice (Oryza sativa L.). Theor. Appl. Genet. 101, 379-387. doi: $10.1007 / \mathrm{s} 001220051494$

Li, Z., Fu, B., Gao, Y., Xu, J., Ali, J., Lafitte, H., et al. (2005b). Genomewide introgression lines and their use in genetic and molecular dissection of complex phenotypes in rice (Oryza sativa L.). Plant Mol. Biol. 59, 33-52. doi: 10.1007/s11103-005-8519-3

Lian, H., Yu, X., Ye, Q., Ding, X., Kitagawa, Y., Kwak, S., et al. (2004). The role of aquaporin RWC3 in drought avoidance in rice. Plant Cell Physiol. 45, 481-489. doi: $10.1093 / \mathrm{pcp} / \mathrm{pch} 058$

Liu, H. Y., Yu, X., Cui, D. Y., Sun, M. H., Sun, W. N., Tang, Z. C., et al. (2007). The role of water channel proteins and nitric oxide signaling in rice seed germination. Cell Res. 17, 638-649. doi: 10.1038/cr.2007.34

Lu, L., Shao, D., Qiu, X., Sun, L., Yan, W., Zhou, X., et al. (2013). Natural variation and artificial selection in four genes determine grain shape in rice. New Phytol. 200, 1269-1280. doi: 10.1111/nph.12430

Nguyen, T., Klueva, N., Chamareck, V., Aarti, A., Magpantay, G., Millena, A., et al. (2004). Saturation mapping of QTL regions and identification of putative candidate genes for drought tolerance in rice. Mol. Genet. Genomics 272, 35-46. doi: 10.1007/s00438-004-1025-5

Ooka, H., Satoh, K., Doi, K., Nagata, T., Otomo, Y., Murakami, K., et al. (2003). Comprehensive analysis of NAC family genes in oryza sativa and arabidopsis thaliana. DNA Res. 10, 239-247. doi: 10.1093/dnares/10.6.239

Palanog, A. D., Swamy, B. P. M., Shamsudin, N. A. A., Dixit, S., Hernandez, J. E., Boromeo, T. H., et al. (2014). Grain yield QTLs with consistent-effect under reproductive-stage drought stress in rice. Field Crop Res. 161, 46-54. doi: 10.1016/j.fcr.2014.01.004

Takasaki, H., Maruyama, K., Kidokoro, S., Ito, Y., Fujita, Y., Shinozaki, K., et al. (2010). The abiotic stress-responsive NAC-type transcription factor OsNAC5 regulates stress-inducible genes and stress tolerance in rice. Mol. Genet. Genomics 284, 173-183. doi: 10.1007/s00438-0100557-0

Venuprasad, R., Bool, M. E., Quiatchon, L., Sta Cruz, M. T., Amante, M., and Atlin, G. N. (2011). A large-effect QTL for rice grain yield under upland drought stress on chromosome 1. Mol. Breed. 30, 535-547. doi: 10.1007/s11032-011-9642-2

Venuprasad, R., Lafitte, H. R., and Atlin, G. N. (2007). Response to direct selection for grain yield under drought stress in rice. Crop Sci. 47:285. doi: $10.2135 /$ cropsci2006.03.0181

Venuprasad, R., Sta Cruz, M. T., Amante, M., Magbanua, R., Kumar, A., and Atlin, G. N. (2008). Response to two cycles of divergent selection for grain yield under drought stress in four rice breeding populations. Field Crop Res. 107, 232-244. doi: 10.1016/j.fcr.2008.02.004

Wang, D., Pan, Y., Zhao, X., Zhu, L., Fu, B., and Li, Z. (2011). Genome-wide temporal-spatial gene expression profiling of drought responsiveness in rice. BMC Genomics 12:149. doi: 10.1186/1471-2164-12-149 
Wang, Y., Zang, J., Yong, S., Jauhar, A., Xu, J., and Li, Z. (2012). Identification of genetic overlaps for salt and drought tolerance using simple sequence repeat markers on an advanced backcross population in rice. Crop. Sci. 52, 1583-1592. doi: 10.2135/cropsci2011.12.062

Wang, W.-S., Zhao, X.-Q., Li, M., Huang, L.-Y., Xu, J.-L., Zhang, F., et al. (2015). Complex molecular mechanisms underlying seedling salt tolerance in rice revealed by comparative transcriptome and metabolomic profiling. J. Exp. Bot. 67, 405-419. doi: 10.1093/jxb/erv476

Wang, Y., Zang, J., Sun, Y., Ali, J., Xu, J., and Li, Z. (2013a). Backgroundindependent quantitative trait loci for drought tolerance identified using advanced backcross introgression lines in rice. Crop Sci. 53, 430-441. doi: 10.2135/cropsci2012.06.0361

Wang, Y., Zhang, L. B., Nafisah, A., Zhu, L. H., Xu, J. L., and Li, Z. K. (2013b). Selection efficiencies for improving drought/salt tolerance yield using introgression breeding in rice (Oryza sativa L.). Crop J. 1, 134-142. doi: 10.1016/j.cj.2013.07.006

Xu, J., Lafitte, H., Gao, Y., Fu, B., Torres, R., and Li, Z. (2005). QTLs for drought escape and tolerance identified in a set of random introgression lines of rice. Theor. Appl. Genet. 111, 1642-1650. doi: 10.1007/s00122-005-0099-8

Yang, W., Kong, Z., Omo-Ikerodah, E., Xu, W., Li, Q., and Xue, Y. (2008). Calcineurin B-like interacting protein kinase OsCIPK23 functions in pollination and drought stress responses in rice (Oryza sativa L.). J. Genet. Genomics. 35, 531-543. doi: 10.1016/S1673-8527(08)60073-9

You, J., Li, Q., Yue, B., Xue, W.Y., Luo, L. J., and Xiong, L. Z. (2006). Identification of quantitative trait loci for $\mathrm{ABA}$ sensitivity at seed germination and seedling stages in rice. Yi. Chuan Xue. Bao. 33, 532-541. doi: 10.1016/S0379-4172(06)60082-6
Yue, B., Xiong, L., Xue, W., Xing, Y., Luo, L., and Xu, C. (2005). Genetic analysis for drought resistance of rice at reproductive stage in field with different types of soil. Theor. Appl. Genet. 111, 1127-1136. doi: 10.1007/s00122-005-0040-1

Yue, B., Xue, W., Luo, L., and Xing, Y. (2008). Identification of quantitative trait loci for four morphologic traits under water stress in rice (Oryza sativa L.). J. Genet. Genomics 35, 569-575. doi: 10.1016/S1673-8527(08)60077-6

Zhang, F., Ma, X. F., Gao, Y. M., Hao, X. B., and Li, Z. K. (2014). Genome-wide response to selection and genetic basis of cold tolerance in rice (Oryza sativa L.). BMC Genet. 15:55. doi: 10.1186/1471-2156-15-55

Zhang, F., Zhai, H.-Q., Paterson, A. H., Xu, J.-L., Gao, Y.-M., Zheng, T.-Q., et al. (2011). Dissecting genetic networks underlying complex phenotypes: the theoretical framework. PLoS ONE 6:e14541. doi: 10.1371/journal.pone.0014541

Zhang, L. (2012). Identification of gene modules associated with drought Response in rice by network-based analysis. PLoS ONE 7:e33748. doi: 10.1371/journal.pone.0033748

Conflict of Interest Statement: The authors declare that the research was conducted in the absence of any commercial or financial relationships that could be construed as a potential conflict of interest.

Copyright (c) 2018 Cui, Zhang, Lin, Xu, Xu and Li. This is an open-access article distributed under the terms of the Creative Commons Attribution License (CC $B Y)$. The use, distribution or reproduction in other forums is permitted, provided the original author(s) and the copyright owner are credited and that the original publication in this journal is cited, in accordance with accepted academic practice. No use, distribution or reproduction is permitted which does not comply with these terms. 